\title{
Messung hoher Elektronendichten mit einer neuartigen Mikrowellen-Reflexionssonde an einem linearen $z$-Pinch ${ }^{*, * *}$
}

\author{
H. Hermansdorfer \\ Institut für Plasmaphysik, Garching bei München \\ (Z. Naturforschg. 21 a, 1471-1478 [1966] ; eingegangen am 3. Juni 1966)
}

\begin{abstract}
A microwave reflection probe method allowing the local measurement of electron densities well above the critical density is described. Measurements in the $35 \mathrm{Gc} / \mathrm{s}$ band on a $z$-pinch show its applicability. A rectangular waveguide with a dielectric aperture window serves as a probe. The interferometer for measuring the reflection coefficient is presented. It indicates phase angle and amplitude automatically without using modulators or frequency translators. Electron densities between about $10^{13} \mathrm{~cm}^{-3}$ and $10^{16} \mathrm{~cm}^{-3}$ could be measured, facilitated by the transforming action of a dielectric $\lambda / 4$-window.
\end{abstract}

Ein magnetfeldfreies Plasma reflektiert elektromagnetische Wellen praktisch vollständig, wenn die Elektronenplasmafrequenz die Wellenfrequenz übertrifft, also wenn die Elektronendichte eine von der Wellenfrequenz abhängige kritische Dichte überschreitet. Im Gegensatz zur Amplitude ist aber der Phasenwinkel einer Welle, die an einem eben begrenzten, homogenen Plasma reflektiert wird, auch weit oberhalb der kritischen Dichte noch gut meßbar von der Elektronendichte abhängig. Für den Modellfall stoßfreier Elektronen, der die Verhältnisse gut beschreibt, ist der Brechungsindex oberhalb der kritischen Dichte rein imaginär. Seine Messung über den Phasenwinkel der reflektierten Welle erlaubt die Bestimmung überkritischer Dichten. Die Eindringtiefe ist klein, wenn die Dichte hoch ist, so daß die Messung praktisch lokal ist.

Die Möglichkeit, Dichten oberhalb der kritischen Dichte über den Reflexionsfaktor des Plasmas für Mikrowellen zu messen, beschrieben erstmals TAKEDA und Mitarbeiter ${ }^{1}$. Sie untersuchten in früheren Arbeiten Plasmen in Hohlleitern und in einer neueren Arbeit ${ }^{2}$ mit einer offenen Sonde ein stationäres Plasma.

In der vorliegenden Arbeit wird neben einem besonders vorteilhaften Meßverfahren die Anwendung von Reflexionssonden auf ein schnell komprimiertes Plasma beschrieben. In Kapitel 1 wird die Reflexion an einer Plasmagrenze modellmäßig behandelt. Der Zusammenhang zwischen den Plasmaeigenschaften und einer senkrecht reflektierten Welle

* Dissertationsauszug, Techn. Hochschule München, 1965.

* Die nachstehende Arbeit wurde im Rahmen des Vertrages zwischen dem Instiut für Plasmaphysik $\mathrm{GmbH}$ und der Europäischen Atomgemeinschaft über die Zusammenarbeit auf dem Gebiete der Plasmaphysik durchgeführt.

1 S. TAKEDA u. T. Tsukishima, J. Phys. Soc. Japan 18, 3 [1963]. wird zunächst für eine Mediengrenze freier Raum - Plasma (Abschnitt 1.1) dann unter Berücksichtigung eines dielektrischen $\lambda / 4$-Fensters (Abschnitt 1.2) erläutert. Ein $\lambda / 4$-Fenster beeinflußt die Dichteempfindlichkeit der Welle günstig. Bei der untersuchten schnellen Entladung war ein Fenster von vorneherein notwendig, um ein unkontrolliertes Eindringen von Plasma in den Hohlleiter zu vermeiden. In Abschnitt 1.3 wird die Berechtigung des Modells für einen Rechteckhohlleiter mit dielektrischem Aperturfenster als Reflexionssonde diskutiert. In Kapitel 2 wird das Mikrowellen-Interferometer beschrieben. Es zeichnet sich dadurch aus, daß es passiv, also ohne Modulation oder Frequenzumsetzer eine eindeutige Messung der Phase und der Amplitude der Meßwelle ermöglicht. Der komplexe Reflexionsfaktor kann damit direkt dargestellt werden. In Kapitel 3 werden Dichtemessungen mit der Reflexionssonde an einem schnellen $z$-Pinch beschrieben. Der zeitliche Dichteverlauf wurde bei verschiedenen Radien im Entladungsvolumen (Abschnitt 3.2) und direkt an der inneren Gefäßwand (Abschnitt 3.5) verfolgt.

\section{Modellmäßige Behandlung der Reflexion an einer Plasmagrenze}

\subsection{Reflexion an einer Mediengrenze Dielektrikum - Plasma}

Für den Plasmabrechungsindex $n_{\mathrm{p}}^{*}$ wird die Beziehung

$$
n_{\mathrm{p}}^{* 2}=\left(\mu_{\mathrm{p}}-i \chi_{\mathrm{p}}\right)^{2}=1-\frac{\left(\omega_{\mathrm{p}} / \omega\right)^{2}}{1-i \cdot \nu / \omega}
$$

verwendet $^{3}$, wobei

2 C. K. McLane, S. TAkeda, W. E. Thomas u. R. C. Thompson, J. Appl. Phys. 36, 2 [1965].

3 J. A. Ratcliffe, The magneto-ionic theory and its application to the ionosphere, Cambridge University Press, London 1959. 
$\mu_{\mathrm{p}}, \chi_{\mathrm{p}}$ Real- und Imaginärteil des Brechungsindex, $\omega_{\mathrm{p}} / 2 \pi \quad$ Elektronenplasmafrequenz,

$\omega / 2 \pi$ Meßfrequenz,

$v$ effektive Stoßfrequenz eines Elektrons.

* bezeichnet Größen mit komplexen Werten.

Es ist im Rahmen dieser Arbeit unbedeutend, daß diese Gleichung für $n_{\mathrm{p}}^{*}$ sehr hohe Dichten unter Umständen etwas zu niedrig wiedergibt ${ }^{4}$.

Die Elektronenplasmafrequenz ist der Wurzel aus der Elektronendichte $N\left[\mathrm{~cm}^{-3}\right]$ proportional:

$$
\omega_{\mathrm{p}} / 2 \pi \cong 9 \cdot 10^{3} \sqrt{N}\left[\mathrm{~s}^{-1}\right] .
$$

Als kritische Dichte $N_{\mathrm{k}}$ bezeichnet man die Elektronendichte, bei der Plasmafrequenz und Meßfrequenz gleich sind. Dann gilt

$$
N / N_{\mathrm{k}}=\left(\omega_{\mathrm{p}} / \omega\right)^{2} .
$$

Unter Verwendung der Beziehung

$$
v=N e^{2} \eta / m
$$

wird für die Darstellung eines sehr großen Dichtebereichs der spezifische Plasmawiderstand $\eta$ der Elektronenstoßfrequenz als Parameter vorgezogen, weil er viel schwächer von der Plasmadichte $N$ abhängt. (Nachdem hier ein quasineutrales Deuteriumplasma betrachtet wird, bezeichnet $N$ auch die Ionendichte.)

Die senkrechte Reflexion an einer Mediengrenze freier Raum - Plasma ist in Abb. 1 skizziert. An

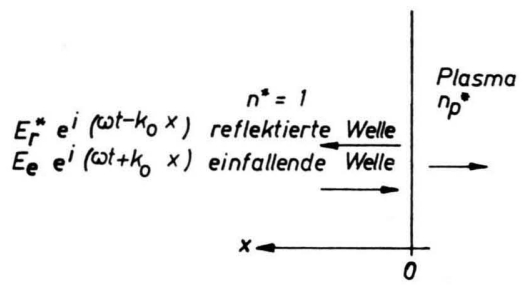

Abb. 1. Senkrechte Reflexion einer ebenen Welle an einer Mediengrenze freier Raum-Plasma.

der Grenzfläche gilt für das Verhältnis $R_{0}{ }^{*}$ der reflektierten zur einfallenden Welle die Fresnelsche Beziehung

$$
R_{0}^{*}=R_{0} e^{i \varphi_{0}}=\frac{E_{\mathrm{r}}^{*}}{E_{\mathrm{e}}}=\frac{1-n_{\mathrm{p}}^{*}}{1+n_{\mathrm{p}}{ }^{*}} .
$$

Dabei ist $\varphi_{0}$ der Phasenwinkel der reflektierten relativ zur einfallenden Welle, $R_{0}$ das Amplitudenverhältnis der beiden Wellen.

\footnotetext{
4 I. P. ShKarofsky, Can. J. Phys. 39, 1619 [1961].
}

Das Polardiagramm Abb. 2 zeigt den Reflexionsfaktor $R_{0}{ }^{*}$ für eine Mediengrenze freier Raum Plasma in Abhängigkeit von der normierten Elektronendichte. Der spezifische Plasmawiderstand erscheint als Parameter. Der schraffierte Bereich entspricht formal negativen Elektronendichten.

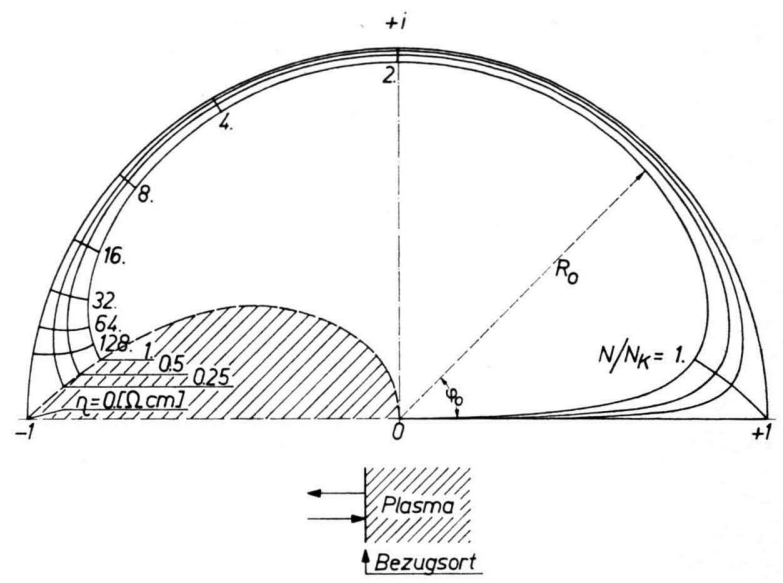

Abb. 2. Reflexionsfaktor $R_{0}^{*}=R_{0} e^{i \varphi_{0}}$ für senkrechte Reflexion an einer Mediengrenze freier Raum-Plasma. Die Abhängigkeit vom spezifischen Plasmawiderstand $\eta$ gilt für die verwendete Meßfrequenz von $35,3 \mathrm{GHz}$.

\subsection{Transformation des Reflexionsfaktors durch ein dielektrisches Fenster}

Wie aus Abb. 2 zu ersehen ist, bedingt ein Meßfehler $\Delta \varphi_{0}$ des Phasenwinkels $\varphi_{0}$ mit steigender Dichte einen immer größeren Fehler der gemessenen Dichte. Die Dichteabhängigkeit des Phasenwinkels $\varphi_{0}$ erfährt in dieser Hinsicht eine günstige Änderung, wenn das dielektrische Sondenfenster, das primär eine scharfe Grenze zum Plasma schaffen soll, als $\lambda / 4$-Fenster dimensioniert wird.

Der Reflexionsfaktor mit dielektrischem Fenster wird wieder für senkrechte Reflexion behandelt. Zunächst wird gezeigt, wie sich der Reflexionsfaktor mit dielektrischem Fenster vorteilhaft nach einer in der Leitungstheorie üblichen Methode ${ }^{5}$ berechnen läßt, bei der das Problem von der generatorabgewandten Seite her aufgerollt wird.

In Abb. 3 sind die Verhältnisse skizziert. An der Grenze Dielektrikum - Plasma bei $x=0$ gilt

$$
R_{0}^{*}=\frac{E_{\mathrm{rd}}^{*}}{E_{\mathrm{ed}}^{*}}=\frac{n_{\mathrm{d}}^{*}-n_{\mathrm{p}}^{*}}{n_{\mathrm{d}}^{*}+n_{\mathrm{p}}^{*}} .
$$

${ }^{5}$ G. KLAGEs, Einführung in die Mikrowellenphysik, Wissenschaftl. Forschungsberichte Bd. 64 [1956], Verlag Steinkopff, Darmstadt. 


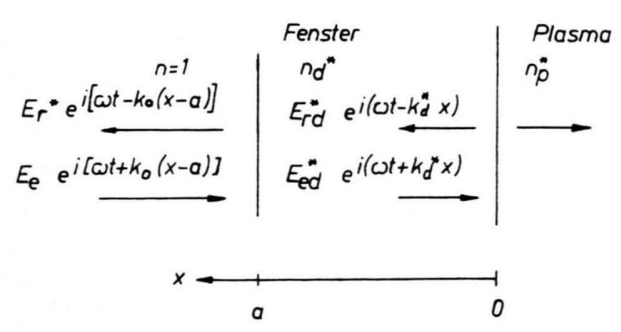

Abb. 3. Senkrechte Reflexion einer ebenen Welle an einem Plasma hinter einem dielektrischen Fenster.

An der Grenzfläche bei $x=a$ ist das Verhältnis der zurückkommenden zur einfallenden Welle

$$
\begin{aligned}
R_{\mathrm{a} 1}^{*} & =\left(E_{\mathrm{rd}}^{*} / E_{\mathrm{ed}}^{*}\right) \exp \left\{-i 2 k_{\mathrm{a}}^{*} a\right\} \\
& =R_{0}^{*} \exp \left\{-i 4 \pi n_{\mathrm{d}}^{*} a / \lambda_{0}\right\} .
\end{aligned}
$$

Mit dem noch zu berechnenden Verhältnis $R_{\mathrm{a}}^{*}=E_{\mathrm{r}}^{*} / E_{\mathrm{e}}^{*}$ gilt wegen des stetigen Übergangs der resultierenden Feldstärken bei $x=a$

$$
\begin{aligned}
& E_{\mathrm{e}}\left(1+R_{\mathrm{a}}^{*}\right)=E_{\mathrm{ed}}^{*} \exp \left\{i k_{\mathrm{d}}^{*} a\right\}\left(1+R_{\mathrm{a} 1}^{*}\right), \\
& H_{\mathrm{e}}\left(1-R_{\mathrm{a}}^{*}\right)=H_{\mathrm{ed}}^{*} \exp \left\{i k_{\mathrm{d}}^{*} a\right\}\left(1-R_{\mathrm{a} 1}^{*}\right) .
\end{aligned}
$$

Nun folgt aus

$$
\operatorname{rot} \mathfrak{F}=-\partial \mathscr{S} / \partial t
$$

für eine fortschreitende ebene Welle

$$
(H / E)^{*}=n^{*} / c \text {. }
$$

Mit $\quad H_{\mathrm{e}} / E_{\mathrm{e}}=1 / c$ und $H_{\mathrm{ed}}^{*} / E_{\mathrm{ed}}^{*}=n_{\mathrm{d}}^{*} / c$

ergibt für das Verhältnis $(H / E)^{*}$ der resultierenden Feldstärken :

$$
\frac{1-R_{\mathrm{a}}^{*}}{1+R_{\mathrm{a}}^{*}}=n_{\mathrm{d}}^{*} \frac{1-R_{\mathrm{a} 1}^{*}}{1+R_{\mathrm{a} 1}^{*}} .
$$

Mit der Abkürzung $n_{f}^{*}=n_{\mathrm{d}}^{*}\left(1-R_{\mathrm{a} 1}^{*}\right) /\left(1+R_{\mathrm{a} 1}^{*}\right)$ folgt endgültig $R_{\mathrm{a}}^{*}=\left(1-n_{\mathrm{f}}^{*}\right) /\left(1+n_{\mathrm{f}}^{*}\right)$. Abb. 4 zeigt eine Polardarstellung des Reflexionsfaktors $R_{\mathrm{a}}^{*}$ für den speziellen Fall einer $\lambda / 4$-Scheibe aus Quarz (geschmolzener Quarz).

Im Grenzfall $\eta=0$ und für $N / N_{\mathrm{k}} \geqq 1$ gilt für den Phasenwinkel $\varphi_{0}$ in Abb. 2

$$
\varphi_{0}=2 \operatorname{arctg} \sqrt{N / N_{\mathrm{k}}-1},
$$

während sich für den Phasenwinkel $\varphi_{\mathrm{a}}$ in $\mathrm{Abb} .4$

$$
\varphi_{\mathrm{a}}=\pi+2 \operatorname{arctg}\left(\sqrt{N / N_{\mathrm{k}}-1} / \mu_{\mathrm{d}}^{2}\right)
$$

ergibt (für $n_{\mathrm{d}}{ }^{*}=\mu_{\mathrm{d}}$ ). Läßt man einen bestimmten prozentualen Fehler der Dichtemessungen zu, so entspricht einer oberen Dichtegrenze $N_{0}$ ohne Fenster die Grenze $N_{\lambda / 4}=\mu_{\mathrm{d}}^{4} N_{0}$ mit $\lambda / 4$-Fenster. Auf diese aus der Leitungstheorie bekannte Möglichkeit, den Reflexionsfaktor zu transformieren, wurde schon von TAKEDA und Mitarbeitern ${ }^{1}$ hingewiesen.

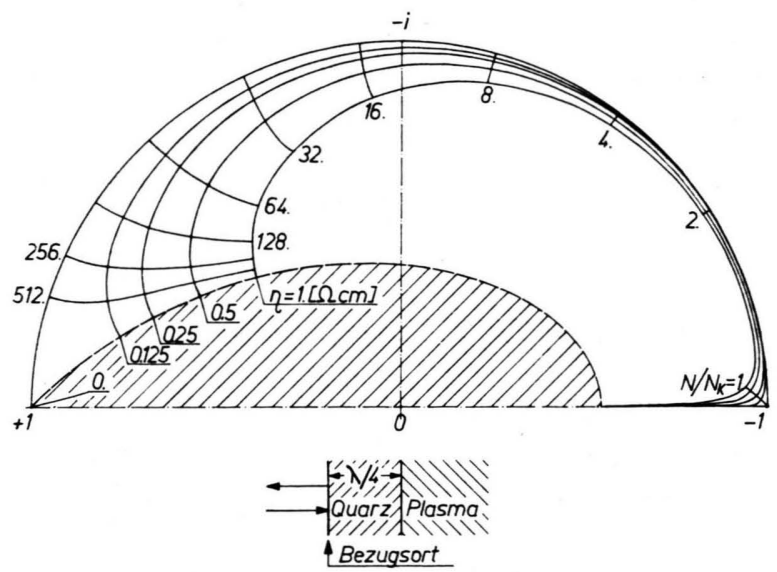

Abb. 4. Reflexionsfaktor $R_{\mathrm{a}}$ * für senkrechte Reflexion an einem Plasma bei Beobachtung durch ein Quarz- $\lambda / 4$-Fenster $\left(\mu_{\mathrm{d}}=1,85\right)$. Die Abhängigkeit vom spezifischen Plasmawiderstand $\eta$ gilt für die verwendete Meßfrequenz von $35,3 \mathrm{GHz}$.

Das Verhalten des Reflexionsfaktors bei Variation der Fensterdicke wird hier nicht näher diskutiert. Der Winkelbereich des Reflexionsfaktors beträgt über $180^{\circ}$, wenn die Scheibendicke etwas kleiner als $\lambda / 4$ ist. Man läßt sich bei der Wahl des Fensters vom interessierenden Dichtebereich leiten. Ist eine weitere Steigerung der Empfindlichkeit erwünscht, so empfiehlt es sich, mehrere $\lambda / 4$-Transformatoren in Serie zu legen.

\subsection{Diskussion des Modells}

Die Behandlung senkrecht reflektierter ebener Wellen zeigte die wesentlichen Züge der Reflexion an dichten Plasmen. Der verwendete $\mathrm{TE}_{10}$-Wellentyp verhält sich bei der Reflexion wie eine schräg einfallende ebene Welle mit dem elektrischen Vektor senkrecht zur Einfallsebene.

Der in Abschnitt 1.2 gezeigte Rechnungsgang zur Ermittlung der reflektierten Welle kann direkt für die Hohlleiterwelle übernommen werden, wenn man alle Brechungsindizes $n^{*}$ durch $\sqrt{\left(n^{*}\right)^{2}-\left(\lambda_{0} / \overline{2 a)^{2}}\right.}$ ersetzt. Dabei ist $a$ die große Seite des Hohlleiterquerschnitts ${ }^{5}$.

In dem damit festgelegten Modell schließt sich im Hohlleiter an das Fenster ein homogenes, magnetfeldfreies Plasma an. Die in diesem Modell steckenden Annahmen sollen einzeln diskutiert werden:

a) Vernachlässigung der Fensterberandung

Nimmt man als Kriterium, daß die Eindringtiefe $D=\lambda_{0} / 2 \pi \chi_{\mathrm{p}}$ deutlich kleiner sein soll als die Quer- 
schnittsabmessungen des Fensters, so ist für Dichten oberhalb einiger $10^{13} \mathrm{~cm}^{-3}$ kein wesentlicher Einfluß der Fensterberandung mehr zu erwarten. Eine heuristische, halbempirische Behandlung des Apertureinflusses findet sich bei TAKEDA u. a. ${ }^{6}$.

b) Annahme eines homogenen Plasmas

Abgesehen von der Raumladungsschicht an der Fensterfläche kann man bei der geringen Eindringtiefe der Welle die Dichte in Richtung der Fensternormalen als konstant ansehen. Eine Abschätzung zeigt, daß Eindringtiefe und Wellenlänge im Plasma durchwegs um Größenordnungen über der DeвyeLänge liegen. Der steile Elektronendichteabfall zur Fensterfläche hin ist praktisch einer scharfen Plasmagrenze gleichwertig. - Die Frage, ob das Plasma im Meßbereich repräsentativ ist, wird im Zusammenhang mit den Messungen diskutiert.

Die radiale Plasmabewegung der Pinchentladung wird möglichst wenig behindert, wenn sie parallel zur Fensterfläche verläuft. Dann muß aber bei der Fensterabmessung von $0,7 \mathrm{~cm}$ in radialer Richtung mit dem radialen Dichtegradienten des Plasmas gerechnet werden, der hauptsächlich eine ungleichmäßige Phase über den Fensterquerschnitt bedingt. Dadurch können im Fenster entgegen den Annahmen höhere Wellentypen angeregt werden.

Eine ungleichmäßige Dichteverteilung über das Fenster wirkt sich mit zunehmender Dichte auf die Phasenverteilung weniger aus. Denkt man beispielsweise an einen Dichteanstieg um den Faktor 2 von Rand zu Rand des Fensters, so nimmt der entsprechende Phasenunterschied mit steigender Dichte ab.

Mildernd ist auch die sinusförmige Verteilung der elektrischen Feldstärke in radialer Richtung über den Fensterquerschnitt. Wenn auch die Phase nicht einfach mit einer sinusförmigen Gewichtsverteilung gemittelt wird, kann man doch sagen, daß der mittlere Teil der Sondenfläche mit hoher Feldamplitude ein größeres Gewicht hat als die Randzonen mit geringer Amplitude.

c) Vernachlässigung des äußeren Magnetfeldes

Nur zum elektrischen Feld der Welle senkrechte Komponenten $B_{\perp}$ des äußeren Magnetfeldes können

6 S. Takeda u. T. Tsukishima, Nat. Bur. Stand. Techn. Note 256 [1965]. die Wellenausbreitung beeinflussen. Als Kriterium dient das Verhältnis der Elektronengyrofrequenz $\omega_{B \perp}$ zur Meßfrequenz $\omega$. Die Bedingung $\omega_{B \perp} / \omega \ll 1$ läßt sich beim linearen $z$-Pinch durch Orientierung des elektrischen Wellenvektors tangential zu dem azimutalen Magnetfeld der Entladung praktisch immer erfüllen. Bei der Meßfrequenz von $35,5 \mathrm{GHZ}$ bedeutet diese Bedingung $B_{\perp} \ll 12,5 \mathrm{kG}$.

\section{Mikrowellen-Interferometer zur Reflexions- messung}

\subsection{Prinzip}

Der komplexe Reflexionsfaktor wurde mit einem passiven Interferometer direkt gemessen. Passiv heißt, daß das Verfahren keine Modulation erfordert; damit begrenzen allein die Mikrowellendetektoren und die Verstärker die Zeitauflösung. Die Grundidee der Messung des komplexen Reflexionsfaktors wurde von KaIser et al. ${ }^{7}$ bereits im Dezimeterbereich angewandt, doch dürfte ihre Durchführung im mm-Gebiet eine nähere Beschreibung wert sein.

Die Meßwelle wird einer vom gleichen Generator abgezweigten Vergleichswelle überlagert. Die Interferenz ist in dem Zeigerdiagramm Abb. 5 dargestellt. Für die resultierende Amplitude $E_{\mathrm{R}}$ gilt

$$
\left.E_{\mathrm{R}}=\sqrt{E_{\mathrm{v}}{ }^{2}+E_{\mathrm{m}}{ }^{2}+2 E_{\mathrm{v}} E_{\mathrm{m}} \cos \left(\varphi_{0}+\varphi_{\mathrm{m}}\right.}\right),
$$

wobei $E_{\mathrm{v}}$ die Amplitude der Vergleichswelle, $E_{\mathrm{m}}$ die Amplitude der Meßwelle, $\varphi_{0}$ ein stationär beliebig einstellbarer Phasenwinkel und $\varphi_{\mathrm{m}}$ der zu messende, vom Meßobjekt aufgeprägte Phasenwinkel ist.

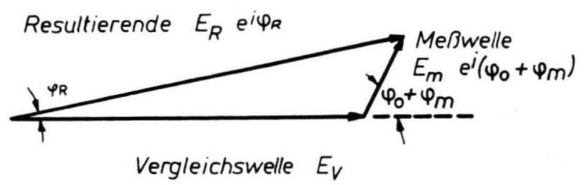

Abb. 5. Zeigerdarstellung der Uberlagerung von Meß- und Vergleichswelle. E Amplitude des elektrischen Feldvektors, $\varphi$ relative Phase.

An symmetrischen Mischern (balanced mixer) lassen sich Meßspannungen

$$
\begin{array}{ll}
U_{1} \sim E_{\mathrm{m}} \cos \varphi_{\mathrm{m}} & \left(\varphi_{0}=0\right), \\
U_{2} \sim E_{\mathrm{m}} \sin \varphi_{\mathrm{m}} & \left(\varphi_{0}=-\pi / 2\right)
\end{array}
$$

erreichen. Um beide Spannungen gleichzeitig zu haben, werden Meß- und Vergleichswelle auf zwei symmetrische Mischer aufgeteilt, wie aus dem Blockschaltbild Abb. 6 zu ersehen ist. Die gewünschten Phasen $\varphi_{0}$ lassen sich mit Phasenschiebern einstellen.

7 J. A. Kaiser, H. B. Smith JR., W. H. Pepper u. J. H. Little, IRE Trans. Microwave Theory Techn. 10, 549 [1962]. 
Eine Darstellung mit $U_{1}$ als Realteil, $U_{2}$ als Imaginärteil ergibt

$$
U_{\mathrm{m}}^{*}=U_{1}+i U_{2} \sim E_{\mathrm{m}} e^{i \varphi_{\mathrm{m}}},
$$

$U_{\mathrm{m}}{ }^{*}$ gibt Phase und Amplitude der Meßwelle linear wieder. Dieses Verfahren eignet sich allgemein für Reflexions- und Transmissionsmessungen.

Durch geeignete Normierung bekommt man aus $U_{\mathrm{m}}{ }^{*}$ den komplexen Reflexionsfaktor. Dazu dient einfacherweise ein Kurzschluß als Meßobjekt (Reflexionsfaktor $\left.R_{\mathrm{K}}{ }^{*}=-1\right)$. Um den Reflexionsfaktor mit der gewünschten Bandbreite von $15 \mathrm{MHz}$ zu messen, sind Meßspannungen von einigen $10 \mathrm{mV}$ vorteilhaft, weil sie sich direkt oszillographisch messen lassen. Für Kristalldioden bedeutet das einen so weiten Arbeitsbereich, daß sich die Bedingung an die Meßspannungen: $U \sim E_{\mathrm{m}} \cos \left(\varphi_{0}+\varphi_{\mathrm{m}}\right)$ nicht mehr durch quadratische Gleichrichtung erreichen läßt. Für die Dioden sind vielmehr ähnliche Betriebsbedingungen wie beim Mischempfang anzustreben, wo einer starken stationären Welle eine schwache Empfangswelle überlagert wird. Bei Strömen um $200 \mu \mathrm{A}$ funktionierten mit $120 \Omega$ Kabelabschlußwiderständen belastete 1N 53B-Mischdioden im gewünschten Sinn.

Für vorbereitende Messungen und zur ständigen Kontrolle werden die Meßspannungen auf einen $x-y$ Oszillographen mit Gleichspannungs-Differenzverstärkern gegeben und so der Reflexionsfaktor polar beobachtet. Zur schnellen Messung im Plasmaexperiment werden die beiden Meßspannungen $U_{1}$ und $U_{2}$ einzeln oszillographiert.

\subsection{Abstimmung}

Störreflexionen im Meßzweig als Folge nicht idealer Bauteile können zu starken Winkelfehlern der gemessenen Welle führen, wenn nämlich Teilwellen mehr- fach am Meßobjekt reflektiert werden. Die in Abb. 6 unter "Reflexionsabstimmung“ zusammengefaßten Elemente dienen der Kompensation störender Reflexionen. Mit dem Abstimmer EH 1 wird zuerst die stationäre, also vom Meßobjekt unabhängige Reflexion im Meßzweig kompensiert (mit einem guten Abschlußwiderstand als Meßobjekt).

Darauf werden mit dem Abstimmer EH 2 Störquellen kompensiert, die eine teilweise Mehrfachreflexion der Meßwelle am Meßobjekt bedingen (bei ausgeschalteter Vergleichswelle darf ein verschiebbarer Kurzschluß als Meßobjekt keine Amplitudenschwankungen mehr hervorrufen). Nach der Abstimmung wird mit einem verschiebbaren Kurzschluß als Meßobjekt das Interferometer auf seine Genauigkeit untersucht. Abb. 7 zeigt das Ergebnis einer Kontrollmessung. Bei einer Winkelgenauigkeit besser als $\pm 3^{\circ}$ wurde die Abstimmung als genügend betrachtet.

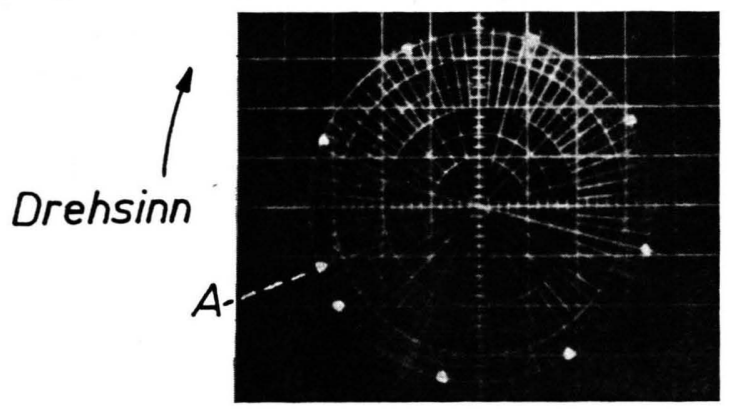

Abb. 7. Kontrolle des Interferometers. Bei der Lage A beginnend wurde der Kurzschluß in 8 gleichen Schritten verschoben.

\subsection{Kontrollmessung des $\lambda / 4$-Fensters}

Um Abweichungen von der idealen Geometrie in ihrer Auswirkung auf die Transformation zu erfassen,

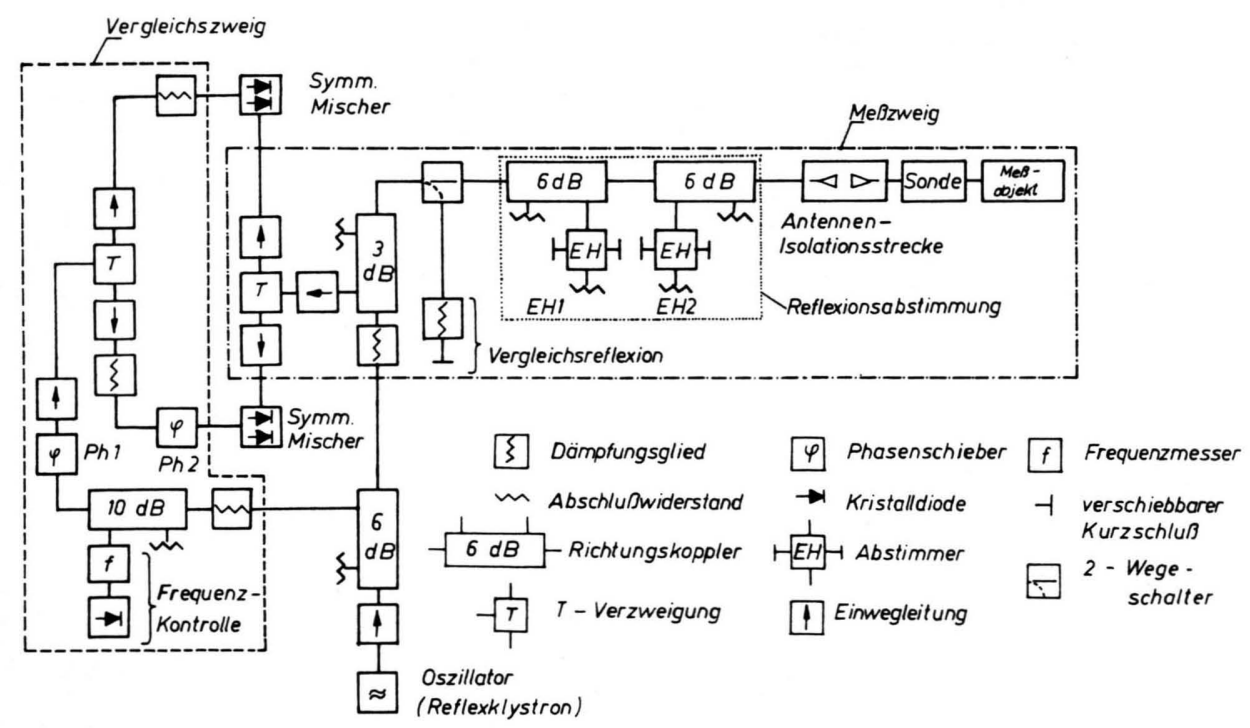

Abb. 6. Blockschaltbild des Mikrowelleninterferometers. Oszillatorleistung ca. $100 \mathrm{~mW}$, Frequenz 35,3 GHz. 
müssen die transformierenden Eigenschaften bei jedem Fenster gemessen werden. Ein Fenster sei als $\lambda / 4$-Fenster dimensioniert. Eine Messung, wie das Fenster die Reflektionsfaktoren +1 und -1 transformiert, führt in einem Iterationsverfahren zu einer genauen Bestimmung der transformierenden Fenstereigenschaften (Brechungsindex, optische Dicke, Absorption), vorausgesetzt, $\mathrm{da} ß$ der $\mathrm{TE}_{10}$-Wellentyp ungestört ist. Der Nachweis dafür ist erbracht, wenn die ermittelten Fenstergrößen die Transformation weiterer Reflexionsfaktoren richtig beschreiben. Bei Quarzfenstern waren die Ergebnisse sehr gut.

\section{Elektronendichtemessungen an einem linearen $z$-Pinch}

Mit der Reflexionsmethode wurden an einem schnellen $z$-Pinch ${ }^{8}$ Dichtemessungen im Entladungsvolumen und an der inneren Gefäßwand durchgeführt. Die Versuchsbedingungen der $z$-Pinch-Entladung sind:

Kondensatorbatterie: $77 \mu \mathrm{F}, 18 \mathrm{kV}, 12,5 \mathrm{~kJ}$

Frequenz des Außenkreises

$106 \mathrm{kHz}$

Gefäßlänge

$50 \mathrm{~cm}$

Gefäßdurchmesser (innen)

$20 \mathrm{~cm}$

Gasfüllung: Deuterium $0,08 \mathrm{~mm} \mathrm{Hg}$

keine Vorionisierung

kein stabilisierendes magnetisches $z$-Feld

\subsection{Anordnung zur Reflexionsmessung}

Abb. 8 zeigt die untersuchten Reflexionsanordnungen:

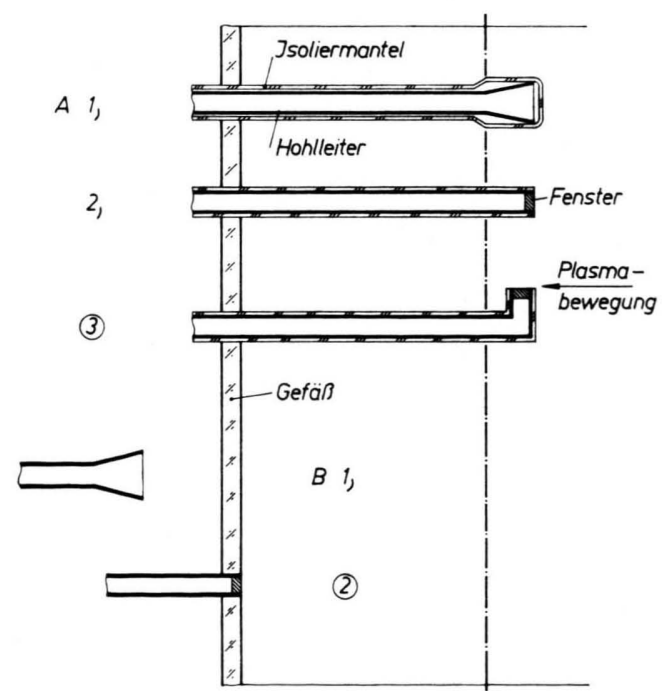

Abb. 8. Untersuchte Reflexionsanordnungen am $z$-Pinch.

8 E. Fünfer u. G. Lehner, Ergeb. Exakt. Naturw. Bd. 34 [1962].
A. Zur Messung im Entladungsvolumen

1. Eine Hornantenne in einem Glasmantel,

2. Eine gerade Sonde mit Fenster, bei der die radiale Plasmabewegung senkrecht zur Fensterfläche verläuft.

3. Eine abgewinkelte Sonde mit Fenster, bei der die radiale Plasmabewegung parallel zur Fensterfläche verläuft.

B. Zur Messung der Restdichte an der Wand

1. Eine Hornantenne außerhalb des Gefäßes,

2. Eine gerade Sonde mit Fenster an der inneren Gefäßwand.

Das Mikrowellen-Interferometer ist so auf einem Schlitten montiert, daß das ganze Mikrowellensystem radialen Verschiebungen der Sonden folgt. Bei allen Anordnungen ist die elektrische Feldstärke der Welle tangential zum azimutalen Eigenmagnetfeld der Entladung orientiert.

Die Anordnungen A 1 und B 1 mit Hornantennen wurden auch für orientierende Versuche mit $4 \mathrm{~mm}$ Wellen verwendet. Die geometrischen Verhältnisse bei Hornantennen beeinträchtigen jedoch die Meßgenauigkeit stark.

Bei der abgewinkelten Sonde A 3 verläuft die radiale Plasmabewegung parallel zur Fensterfläche, ohne daß auf den Vorteil, die Sonde radial verschieben zu können, verzichtet werden muß. Für schwere Teilchen, die aus der Fensterfläche stammen, ist die Impulsübernahme vom Plasma erschwert, sie neigen dazu, in Sondennähe „hängenzubleiben“. Verdampfung des Fenstermaterials wird die Messung zeitlich begrenzen.

Es werden nur Messungen mit den Anordnungen A 3 und B 2 unter Verwendung von Quarz- $\lambda / 4$-Fenstern ausführlicher beschrieben. Die Ergebnisse werden durch die Erfahrungen mit den anderen Anordnungen voll unterstützt. Obwohl die einzelnen Sondentypen die Teilchenbewegung voneinander verschieden behindern, zeigten sie qualitativ den gleichen Zeitverlauf der Dichte. Beobachtungen von EutoN ${ }^{9}$ an sehr energiereichen Entladungen zeigten eine starke Ionisation der Luft außerhalb von Quarzgefäßen. Es ist darauf zu achten, daß nicht ionisierende Strahlung die Luft im Hohlleiter in störendem Maß ionisiert, was eine Winkeländerung des Reflexionsfaktors im gleichen Sinn wie eine Dichteerhöhung des Plasmas zur Folge hätte. Die bei sehr hohen Dichten mit nahezu metallischer

9 R. C. Elton, J. Nucl. Energy C 6, 401 [1964]. 
Reflexion gemessenen Reflexionsfaktoren zeigen, daß eventuelle Winkelabweichungen durch Ionisation im Hohlleiter innerhalb der interferometrischen Fehlergrenzen liegen.

Eine weitere Fehlerquelle ist die Abkühlung des Plasmas an der Sonde. Bei Druckausgleich mit dem heißen ungestörten Plasma steigt die Teilchendichte im Maß der Temperaturabnahme. Solange das heiße Plasma die Sonde schnell überströmt, sollte die Abkühlung erträglich bleiben.

Unter Verwendung von Quarz- $\lambda / 4$-Fenstern konnten Dichten von etwa $10^{13} \mathrm{~cm}^{-3}$ bis $\mathrm{zu}$ einigen $10^{16} \mathrm{~cm}^{-3}$ gemessen werden. Die untere Grenze ist durch die kritische Dichte für $8,5 \mathrm{~mm}$-Wellen gegeben. Die obere Grenze ist allein eine Frage der Meßgenauigkeit. Die Meßgenauigkeit war hauptsächlich vom mechanischen Aufbau und von der Elektronik her beschränkt. Für einigermaßen verläßliche Aussagen über den Plasmawiderstand ist die Meßgenauigkeit zu gering.

\subsection{Dichtemessungen im Entladungsvolumen}

In Abb. 9 ist der mit der abgewinkelten Sonde ermittelte zeitliche Dichteverlauf bei den Radien $r=0,2,4,6$ und $8 \mathrm{~cm}$ dargestellt. Der Winkelfehler der interferometrischen Messung beträgt maximal $6^{\circ}$, dem entsprechen die eingezeichneten mög-

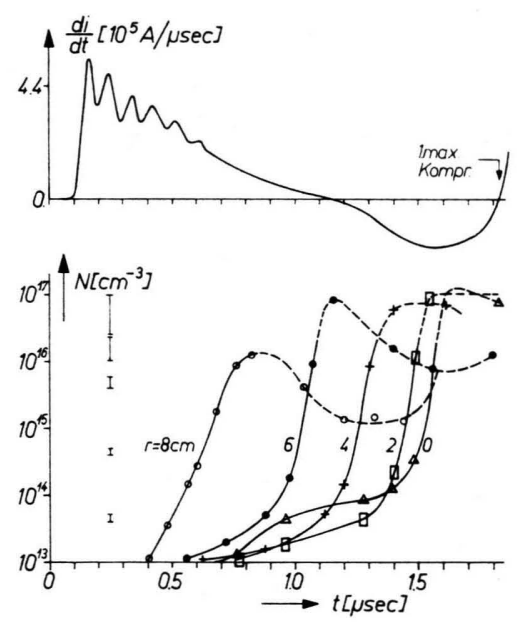

Abb. 9. Zeitverlauf der Stromableitung $\mathrm{d} i / \mathrm{d} t$ und der Dichte $N$ bei verschiedenen Radien $r_{0}=0 \pm 0,5 \mathrm{~cm}, r_{1}=r_{0}+2 \mathrm{~cm}$, usw.

lichen Fehler der Dichtemessung. Ein interferometrischer Meßfehler bewirkt, daß die Dichten systematisch entweder zu hoch oder zu niedrig erscheinen.

Die Diskussion des Dichteverlaufs nach Abb. 9 soll auf die wesentlichen Züge beschränkt sein:
Das an Pinch-Entladungen allgemein beobachtete Schnellerwerden der Kontraktion ist eine Folge der Zylindergeometrie.

Das früh in der Gefäßachse (Kurve mit $r=0$ ) erscheinende relative Dichtemaximum deckt sich weder mit der Vorstellung adiabatischer Kompression noch mit dem Modell einer scharfen Stoßfront. Die Geschwindigkeit der komprimierenden Plasmaschicht ist groß gegenüber wahrscheinlichen Schallgeschwindigkeiten im vorerst wenig ionisierten Innenraum. Die relativ große freie Weglänge und damit die große zu erwartende Schichtdicke im Innenraum ist eine naheliegende Erklärung für den schnellen Ausläufer der Plasmabewegung, der bei der Achse aufgestaut wird. Hier erweist sich die hohe Empfindlichkeit der Reflexionssonde bei relativ niederen Dichten als vorteilhaft.

Im Modell einer sehr dünnen stromführenden Schicht erreicht die komprimierende Schicht nahe beim 2. Nulldurchgang der zeitlichen Änderung des Entladungsstromes $\mathrm{d} i / \mathrm{d} t$ ihren kleinsten Achsenabstand. Dagegen zeigt die Sondenmessung deutlich zuvor ein Maximum der Elektronendichte in der Gefäßachse. Das kann nur so gedeutet werden, daß in der Achse das Plasma bereits reflektiert wird, während die stromführende Schicht noch weiter nach innen läuft. Numerische Rechnungen von HaIN und Mitarbeitern ${ }^{10}$ zeigen, daß bei einem schnellen $z$ Pinch die maximale Kompression nicht durch einen scharfen Zeitpunkt definiert werden kann. Die Rechnungen ergaben bei der 1. max. Kompression deutlich zwei Bereiche mit verschiedenen Vorzeichen der Plasmageschwindigkeit. Damit ist die hier beobachtete Erscheinung qualitativ im Einklang.

Die Kurven für den zeitlichen Dichteverlauf sind teilweise gestrichelt gezeichnet. Dann läßt der bei hohen Dichten möglicherweise große systematische Fehler nur noch qualitative Aussagen zu oder es wird damit gerechnet, daß der Verlauf deutlich durch verdampftes Fenstermaterial mitbestimmt wird.

\subsection{Dichtemessung an der inneren Gefäßoberfläche}

Die Reflexionsanordnung B 2 in Abb. 9 ist zur Messung der Restdichte an der Gefäßwand besonders geeignet. Die Meßergebnisse werden anhand der Dichteauswertung in Abb. 10 diskutiert.

Zunächst fällt auf, daß die Dichte später über $10^{13} \mathrm{~cm}^{-3}$ ansteigt, als etwa bei $r=8 \mathrm{~cm}$. Die Ioni-

10 K. Hain, G. Hain, K. V. Roberts u. W. Köppendörfer, Z. Naturforschg. 15 a, 1039 [1960]. 


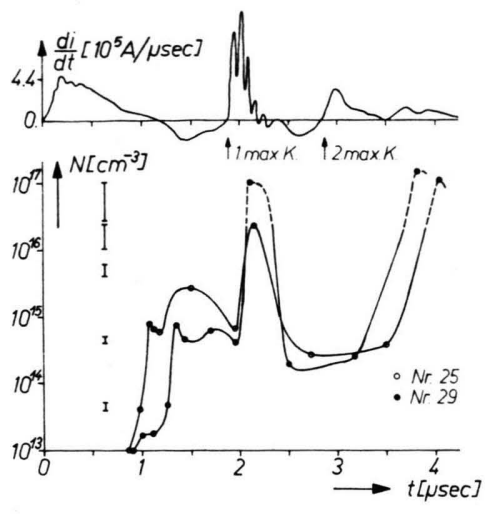

Abb. 10. Zeitlicher Dichteverlauf an der inneren Gefäßwand für zwei verschiedene Entladungen und $\mathrm{d} i / \mathrm{d} t$-Verlauf zur zeitlichen Zuordnung.

sation ist nahe der Wand behindert und führt so erst später zu einer Dichte über $10^{13} \mathrm{~cm}^{-3}$.

Besonders prägnant ist der starke Dichteanstieg kurz nach dem 2. Nulldurchgang von $\mathrm{d} i / \mathrm{d} t$. Diese Erscheinung wird dem auch von anderer Seite vielfach beobachteten Auftreten von Wandströmen zugeschrieben. Als Ursache wird dafür allgemein Strahlung aus der Pinchsäule, im wesentlichen von Verunreinigungen, bei der ersten maximalen Kompression angenommen ${ }^{11}$. Die Entladung im Wandmate- rial, einmal ausgelöst, kann schnell anwachsen. Trotz der hohen Dichte reicht die Leitfähigkeit hier nicht aus, den $\mathrm{d} i / \mathrm{d} t$-Verlauf stark zu beeinflussen.

Es sind Messungen für zwei verschiedene Entladungen gezeigt. Mit zunehmender Entladungszahl wird der Dichteanstieg durch Wandströme zeitlich schärfer ausgeprägt. - Orientierende Versuche mit kleinerem Fülldruck ließen deutlich eine nachfolgende Bewegung der an der Wand erzeugten Plasmaschicht zur Achse erkennen.

Der Dichteanstieg nach 3,5 $\mu$ sec wird durch Wandberührung des Hauptplasmas hervorgerufen.

Zusammenfassend läßt sich sagen, daß trotz der Beschränkungen, die allen Sonden auferlegt sind, bei denen das Plasma nahe an Festkörperflächen gemessen wird, so z. B. auch Langmuir-Sonden, die neuartige Mikrowellenreflexionssonde in der Lage ist, auch bei hohen Anforderungen interessante Vorgänge zu messen oder zumindest deutlich aufzuzeigen. Wegen der kleineren Querschnittsabmessungen wäre der Vorstoß zu $4 \mathrm{~mm}$-Wellen, der möglich sein sollte, zu empfehlen.

Mein Dank gebührt besonders Herrn Prof. Dr. E. FüNFER für die großzügige Förderung dieser Arbeit. Die gute Zusammenarbeit am Institut für Plasmaphysik möchte ich anerkennend erwähnen.

11 H. A. B. Bodin, A. A. Newton u. N. J. Peacock, Nucl. Fusion 1, 54, [1960]. 